\title{
Extracting Heart Rate Variability: A Summary of Camera Based Photoplethysmograph
}

\author{
Rejwana Islam, Mousumi Sarmin, Farzana Akter Dulia, and Moinul Islam Sayed
}

\begin{abstract}
Heart rate is one of the major indicators of our physiological state. An irregular or rapid heartbeat, fainting, dizziness, chest pain or shortness of breath can be found by it. The traditional heart rate observing methods such as electrocardiogram (ECG) require physical contact in order to show the heart rate reading exactly but this is uncomfortable for regular monitoring. Techniques for measuring physiological parameters remotely from hospital, as well as monitoring patients continuously, have been one of the major concerns of the scholars. Many heart rate measurement methods using smartphone, webcam, commercial camera etc. have been proposed by many researchers. Image or video processing is the fundamental technique for measuring heart rate through smartphone. With the aim of exploring different heart rate monitoring methods and the advantages and disadvantages, the present study consulted secondary sources like published articles.
\end{abstract}

Index Terms - Heart rate, Smartphone, Camera, Webcam, Photoplethysmograph.

\section{INTRODUCTION}

The number of times heart beats per minute is known as heart rate, which represents the heart conditions. Heart rate is an important indicator of a person's physiological state and significantly represents its vital signs which are used for basic functionality measurement of the body. Heart Rate Monitor plays an important role in order to show human's fitness level. The normal range of heart rate is $60-90$ beats per minute (bpm).

The traditional heart rate observing method requires physical contact in order to show the heart rate reading exactly but it is uncomfortable for them. Because some of the patients feel allergic and discomfort when a device is attached on their skin. Besides, the attachment of contact heart rate device will restrict movement of patients. For example, the contact heart rate monitor is hard to apply on children and babies since the babies always roll on the bed while the children are hard to settle down themselves.

Contactless heart rate measurement would also be useful

Published on April 26, 2020.

Rejwana Islam, Department of Computer Science and Engineering, Global University of Bangladesh, Bangladesh

(e-mail: rezwanamum@yahoo.com)

Mousumi Sarmin, Faculty of Computer Science and Engineering,

Patuakhali Science and Technology University, Bangladesh.

(e-mail: mousumisarmin@gmail.com)

Farzana Akter Dulia, Faculty of Computer Science and Engineering,

Patuakhali Science and Technology University, Bangladesh.

(e-mail: duliaamin@gmail.com)

Moinul Islam Sayed, Department of Computer Science and

Information Technology, Patuakhali Science and Technology University, Bangladesh.

(corresponding e-mail: smoinul@pstu.ac.bd) to measure the Heart Rate of patient whose conditions may get worse by contact sensors such as burn victims and babies. Contactless heart rate measurement methods like smartphone apps, webcams, consumer cameras etc. are easy to use and the patients do not feel uncomfortable as the physical contacts are not required.

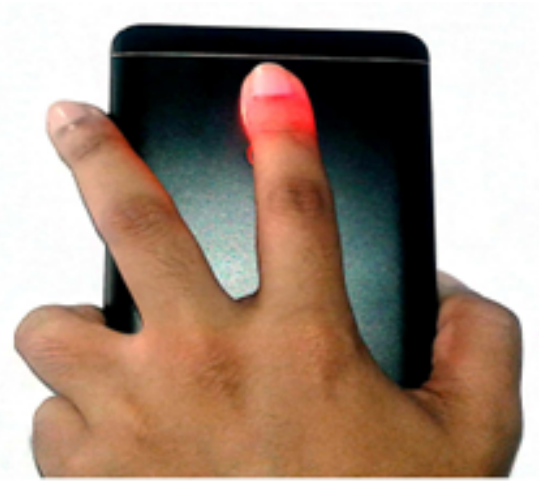

Fig. 1. Heart rate (HR) measurement using smartphone.

At present heart disease patients are increasing in tremendous rate. Heart rate is one of the most important parameters which gives information about health conditions, because it reflects the physical and emotional activities for example, exercise, emotion changes, illness, etc. The objectives of this research are to (a) provide an overview of research conducted for monitoring heart rate, (b) measure heart rate under different distances and illuminations, (c) assess the efficiency of the existing methods, (d) classify published studies by their choice of algorithms and contributions.

\section{LITERATURE REVIEW}

For both commercial and academic purposes HR measurement is an attractive topic. For recording important parameters like heart rate mobile camera can be used effectively. Red and blue constituents are separated. The variation of received intensity of red color with time is shown by the red DC mean(mr) data values, which is used to calculate the heart rate [1]. Fingertip video recorded from smartphone camera is used for measuring heart rate. For the calculation of the PPG values red channel values will be used, because it remains significantly invariant across different smartphones [3].

Hue can be used to measure heart rate [6]. In the proposed iPPG (image PPG) approach average fluctuations of Hue values are measured. Each RGB (Red Green Blue) pixel are convert to the equivalent HSV (Hue Saturation Value) pixel. Then the average Hue value for each frame is computed. Different Hue values represent different colors, for example 
0 represents red, green is indicated by 0.33 , and blue is represented by 0.66 . Using iPPG obtained from the forehead region, Green channel, and Saturation (HSV colorspace) the $\mathrm{HR}$ and RR is computed by the MATLAB script.

The accuracy of HR detection is improved by a machine learning approach [9]. Three raw traces in selected windows were pre-processed and using ICA (Independent Component Analysis) based on the Joint Approximate Diagonalization of Eigenmatrices (JADE) decomposed into three
[17]. The framework is tested on a huge complicated and public database MAHNOB-HCI. Three major processes are contained in the proposed framework: first, Discriminative Response Map Fitting (DRMF) is employed to find the particular face ROI and use tracking for addressing the inflexible head movement problem; second, the interferences of illumination variations is rectified by employing Normalized Least Mean Squares (NLMS) adaptive filter; third, in order to reduce the noise caused by

Table I: Summary of smartphone camera-based methods for heart rate (HR) measurement.

\begin{tabular}{llll}
\hline \hline Ref. no. & Light source & Attachment & Remarks \\
\hline$[1]$ & White light & Face & Results looks very promising. \\
\hline$[2]$ & LED flash & Index finger & Low cost. \\
\hline$[3]$ & With LED \& without LED & Fingertip & $\begin{array}{l}\text { Hough transform cannot be used, radius of ROI method can only be } \\
\text { used on No-LED data. }\end{array}$ \\
\hline$[6]$ & Flash on \& flash off & Face(forehead) & The accuracy of the HR is increased by the additional illumination. \\
\hline$[7]$ & $\begin{array}{l}\text { Independent of constant } \\
\text { illumination. }\end{array}$ & Skin & Result is simple, robust and computationally efficient. \\
\hline$[16]$ & Ambient light & Index finger & Provide satisfactory accuracy, error rate 4.88\%. \\
\hline$[17]$ & Ambient & Face & $\begin{array}{l}\text { Find precise face ROI, reduce the noise, under realistic HCL situations } \\
\text { improve the accuracy of the result. }\end{array}$ \\
\hline$[27]$ & Flash/very well-lit condition & $\begin{array}{l}\text { Index finger } \\
\text { /face }\end{array}$ & Contact methods give accurate results than non-contact methods. \\
\hline \hline
\end{tabular}

independent source signals. To estimate HR from the computed values k-nearest neighbor $(\mathrm{kNN})$ classifier and Linear regression have been used. HR can be measured from online videos. [10]. The average optical intensity changes in the RGB green channel over multiple frames indicates the heartbeat and allows for an estimate of HR. The strongest plethysmographic signal is featured by the green channel. The Fast Fourier transformation is used to transform the green channel into the frequency spectrum. A well-detected $\mathrm{HR}$ is represented by a single distinct peak in the frequency distribution and it has the highest probability to be the correct HR.

Webcam is used to measure heart rate [14]. Blood circulation causes facial skin color variation, from which heart rate can be obtained. Fast Fourier Transform (FFT), Independent Component Analysis (ICA) and Principal Component Analysis (PCA) signal processing methods have been applied on the color channels. Based on the joint approximate diagonalization of Eigen matrices (JADE) algorithm the normalized raw traces were decomposed into three independent source signals ( $R, G$ and $B)$ for the ICA method. Using the Fast Fourier Transform the power spectrum is obtained. A Graphical User Interface (GUI) has been developed using MATLAB to monitor HR in real time.

Face tracking and Normalized Least Mean Square adaptive filtering methods are used to measure heart rate sudden non-rigid movements signal segments with big SD values are discarded.

Another HR measurement method is proposed which is robust to illumination changes, movement, and environment's background is not needed [20]. In case of PG signal extraction, the proposed algorithm can select good local regions. Despite illumination variations the PG signal could be extracted from two raw point traces in the green channel.

For non-contact monitoring of breathing and heart rate estimation Microsoft (MS) Kinect sensors are used to detect possible health and neurological problems [24]. Denoising, spectral analysis and visualization are used by this methodology to determine specific biomedical features. To detect the heart rate the band-pass filtering infinite impulse response (IIR) is applied to extract frequency components.

\section{Methodology}

Some research papers are randomly selected on heart rate measurement. Then these research papers are categorized based on different heart rate monitoring methods followed by the studied research papers. There have been many developments in measuring heart rate using different techniques. Many methods are available such as PPG based methods, contact methods, non-contact method etc. Good results are provided in certain conditions. A comparative

Table II: Summary of webcam-based methods for heart rate (HR) measurement.

\begin{tabular}{llll}
\hline \hline Ref. no. & \multicolumn{1}{c}{ Light source } & Attachment & Remarks \\
\hline$[4]$ & Infrared & Face & $\begin{array}{l}\text { Low cost, touchless HR monitoring to new scenarios such as } \\
\text { night driving. }\end{array}$ \\
\hline$[8]$ & Indirect sun light or fluorescent light & Face & Provides better result under fluorescent light and indirect sunlight. \\
\hline$[10]$ & Sufficient light & Face(forehead) & Provides better result. \\
\hline$[13]$ & RCLED light, ambient light & Face(forehead) & This process takes minimum time and cost. \\
\hline$[14]$ & Ambient sunlight & Face & Easy to implement, low cost, comfortable. \\
\hline$[19]$ & Ambient light & Fingertip & Remove low frequency component \\
\hline$[22]$ & Ambient light & Skin & Low cost, wide range of applications systematic literature review. \\
\hline$[9]$ & $\begin{array}{l}\text { Normal indoor environment, ambient artificial } \\
\text { fluorescent light }\end{array}$ & Face & Accuracy of estimation is increased. \\
\hline
\end{tabular}


study on different types of methods for monitoring heart rate is given. photoplethysmographic signals, the method uses two orthogonal vectors of RGB color space. To reduce artifacts, denoising algorithm based on dual tree complex wavelet

Table III: Comparison between heart rates measured by the electrocardiogram and those measured by the smartphone using the five algorithms for selection of region of interest.

\begin{tabular}{cccc}
\hline \hline Algorithm & $\mathrm{r}$ & SEE (bpm) & \multicolumn{2}{c}{ Bland-Altman analysis } \\
\cline { 2 - 4 } & & & 2.45 \\
\hline VAR vs ECG & $0.975(\mathrm{p}<0.001)$ & 4.65 & -0.34 \\
\hline SER vs ECG & $0.913(\mathrm{p}<0.001)$ & 1.49 & -0.59 \\
\hline TM vs ECG & $0.991(\mathrm{p}<0.001)$ & -0.31 & -0.29 \\
\hline TD vs ECG & $0.992(\mathrm{p}<0.001)$ & 1.40 & 2.89 \\
\hline GRAD vs ECG & $0.971(\mathrm{p}<0.001)$ & 2.51 & -0.25 \\
\hline \hline
\end{tabular}

Note: r: Pearson correlation coefficients; SEE: standard error of estimate; LA: limit of agreement.

Source: Rong, Wen, Ning. Wan, Xiao and Yuan, 2015.

\section{FINDINGS AND Discussion}

Different heart rate monitoring methods are discussed here.

\section{A. Using Smartphone Camera}

By using the camera of a smartphone heart rate variability can be measured, which provides promising result [1]. A method for the heart rate measurement using fingertip video recorded by the camera of a smartphone is proposed [3]. The iPPG method is also used where both heart rate and respiratory rate (RR) can be measured from a subject's face video by monitoring variations in color of reflected light i.e. Hue [5].

A more accurate readings than the Green channel is provided by the procedure of measuring iPPG using Hue (range 0-0.1) gives. The face videos with additional illumination provide better results.

Limitations:

- None of the algorithms is perfect or error-free. A manual correction is usually required by visual inspection on the computer screen, which is time consuming.

- PPG measurements are very sensitive to subject's tissue and movement artifacts.

- Verifying correct usage of camera under good lighting conditions is difficult task.

- In case of all real-world scenarios the algorithms will not work properly.

Summary of smartphone camera-based methods for HR measurement are given in table I.

\section{B. Webcam based}

A machine learning approach using webcam was proposed which improves the accurateness of HR monitpring in naturalistic dimensions [9]. The best result is produced by the third component among all three components among eight out of ten participants by comparing Root Mean Squared Error (RMSE). For automated heart rate (HR) computation from digital color video recordings using a simple webcam is proposed [8]. For extracting transform is used. In-situ opportunities can be established and predicted for webcam-based heart rate monitoring in the environment of the workplace. The data can be analyzed from unobtrusive sensors in a universal manner [19]. They found that whenever, there is limited motion, the number of opportunities are much higher at $86 \%$ compared to $50 \%$ while not considering motion. These opportunities can be predicted with the help of alternate unobtrusive sensing mechanism (mouse, keyboard, system usage etc.) with an accuracy of $81 \%$.

Limitations:

- Although the accuracy of estimation is increased, for all cases (e.g. emergency severity index (ESI) triage) the accuracy is not good enough.

- In case of anemic patients, the error rates are higher than others.

Face region detection, does not work in presence of motion, and require sufficient light/illumination on the facial region are some practical challenges. Summary of some webcambased methods for HR measurement are given in table II.

\section{Different Algorithms}

Smartphone photoplethysmography is a technique which can detect a number of physiological parameters from the photoplethysmographic signal. The signal is obtained by the built-in camera of a smartphone. This method is simple, inexpensive, and user-friendly and can be used in remote medicine and home healthcare service.

For extracting photoplethysmographic signals from the camera the determination of the optimal region of interest (ROI) is an essential issue. Five algorithms were proposed for ROI selection: variance (VAR), spectral energy ratio (SER), template matching (TM), temporal difference (TD), and gradient (GRAD) [24]. The TM and the TD algorithms had less standard error of estimate $(<1.5 \mathrm{bpm})$ and smaller limits of

agreement $(<3 \mathrm{bpm})$, , so that they outperformed the other three. The TD algorithm was a little better than the TM algorithm. To promote the achievability of the sPPG (Smartphone PPG) technique TD algorithm can be used in

Table IV: Comparison of heart rate by four smartphone apps and clinically accepted pulse oximetry compared to electrocardiogram.

\begin{tabular}{ccc} 
Table IV: Comparison of heart rate by four smartphone apps and clinically accepted pulse oximetry compared to electrocardiogram. & \multicolumn{1}{c}{ Pean absolute error (SD) } \\
\hline \hline Measurement method & $4.52(1.12)^{*}$ & 0.83 \\
\hline Instant Heart Rate (IHR) & $1.96(0.48)^{*}$ & 0.96 \\
\hline Heart Fitness (HF) & $7.08(1.39)^{*}$ & 0.62 \\
\hline Whats My Heart Rate (WMH) & $8.11(1.38)^{*}$ & 0.61 \\
\hline Cardiio (CAR) & $2.0(0.35)^{*}$ \\
\hline Pulse oximetry & 0.92 \\
\hline \hline
\end{tabular}


smartphones. Comparative results of heart rate measurement using algorithms for selection of region of interest are given in table III.

\section{Utilising Smartphone Apps}

Mobile health monitoring technology is provided by smartphone manufacturers to their clients. Using the built-in camera and apps heart rate can be measured. The diagnostic
PPG-based apps had higher viability and better accuracy than the two non-contact PPG-based apps for heart rate monitoring.

\section{E. RGB Color Component}

Using the smart phone camera video image of the fingertip is collected. A better insight of the signal is given by the PCA analysis of the RGB color space of the video image

Table V: Summary of RGB based non-contact methods for HR measurement.

\begin{tabular}{|c|c|c|c|}
\hline Reference no. & Camera & Raw signal & Remarks \\
\hline [5] & $\begin{array}{l}\text { Consumer grade } \\
\text { camera }\end{array}$ & Red & Error fall under the standard allowable error limits \\
\hline$[6]$ & Smartphone & Hue & Hue gives more precise results than the green channel \\
\hline [7] & $\begin{array}{l}\text { Digital camera, } \\
\text { smartphone }\end{array}$ & Red and Green & Semi-automated, theoretically not sensitive to illumination \\
\hline [8] & Webcam & Blue and Red & Average error $<3.5 \%$ \\
\hline [9] & Webcam & Red, Green, Blue & RMS error is reduced from $43.76 \mathrm{bpm}$ to $3.64 \mathrm{bpm}$ \\
\hline$[10]$ & Webcam & Green & Feasible to measure HR in online crowdsourced studies \\
\hline$[11]$ & Webcam & Red, Green, Blue & Chrominance feature reduce the color channel magnitude difference \\
\hline [12] & Webcam & Red, Green, Blue & Outperforms the ICA based methods \\
\hline [13] & Webcam & Green & Average accuracy is 95.59083 \\
\hline [14] & Webcam & Red, Green, Blue & Easy to implement, for real time applications \\
\hline$[15]$ & RGB video camera & Red and Green & $\begin{array}{l}\text { Found to be the best performing of the three possible two-signal } \\
\text { combinations }\end{array}$ \\
\hline [17] & iSight camera & Green & $\begin{array}{l}\text { The interference of illumination is reduced by using the green value of } \\
\text { background }\end{array}$ \\
\hline$[21]$ & CCD camera & Green & Conceivable minimum time and cost \\
\hline$[22]$ & Webcam & Red, Green. Blue & Low cost \\
\hline
\end{tabular}

accuracy of different heart rate monitoring apps are tested in medical practice [25]. The achievability and accurateness of measuring heart rate was tested on four apps using both iPhone 4 and

iPhone 5 which are commercially available. 'Instant Heart Rate' (IHR) and 'Heart Fitness' (HF) work with contact photoplethysmography (contact of fingertip to built-in camera), while 'Whats My Heart Rate' (WMH) and 'Cardiio Version' (CAR) work with noncontact photoplethysmography. The results were compared to the heart rate of the pulse oximetry and electrocardiogram. The accuracy of heart rate measured by the smartphone apps are compared to electrocardiogram, reported as mean absolute error (in bpm standard error). The results of four apps have considerable performance differences. The two contact
[28]. Using time frequency transformation, the most variability is contained by the Green band [30]. The second component is used by Poh et al. Several other authors adopted this method [34], [35], [36]. For the FFT method the value of $R, G$ and $B$ are needed. So, $R, G$ and $B$ signals were computed. Based on the joint approximate diagonalization of Eigen matrices (JADE) algorithm the normalized raw traces were decomposed into three independent source signals (R, G and B) [14]. Two input signals $\mathrm{R}$ and $\mathrm{G}$ video signals are used [15].

\section{F. PPG Methods}

Photoplethysmography (PPG) is one of the useful methods which is used in contactless heart rate monitoring. PPG

Table VI: Summary of PPG based methods for HR measurement.

\begin{tabular}{|c|c|c|c|c|c|}
\hline $\begin{array}{l}\text { Reference } \\
\text { no. }\end{array}$ & Light source & Camera & Attachment & Contact/non-contact & Remarks \\
\hline$[8]$ & Ambient & Webcam & Face & Non-contact & $\begin{array}{l}\text { Under fluorescent light and indirect sunlight } \\
\text { provide satisfactory results. }\end{array}$ \\
\hline [9] & $\begin{array}{l}\text { Indoor } \\
\text { environment }\end{array}$ & Webcam & Face & Non-contact & Accuracy of estimation is increased. \\
\hline$[10]$ & Sufficient light & Webcam & Face & Non-contact & Gives more precise results. \\
\hline [13] & Ambient light & Webcam & Forehead & Non-contact & This process takes minimum time and cost. \\
\hline$[15]$ & Ambient & $\begin{array}{l}\text { RGB video } \\
\text { camera }\end{array}$ & Face & Non-contact & $\begin{array}{l}\text { A powerful methodology to deal with noisy, } \\
\text { non-stationary biosignals. }\end{array}$ \\
\hline$[16]$ & Ambient & Smartphone & Index finger & Contact & Error rate $4.88 \%$ \\
\hline [18] & LED & Smartphone & Finger or earlobe & Contact & Positive correlation between HR. \\
\hline$[21]$ & Infrared light & CCD camera & Face & Non-contact & Conceivable minimum time and cost. \\
\hline [22] & Ambient light & Webcam & Skin & Non-contact & $\begin{array}{l}\text { Low cost, wide range of applications } \\
\text { systematic literature review. }\end{array}$ \\
\hline
\end{tabular}




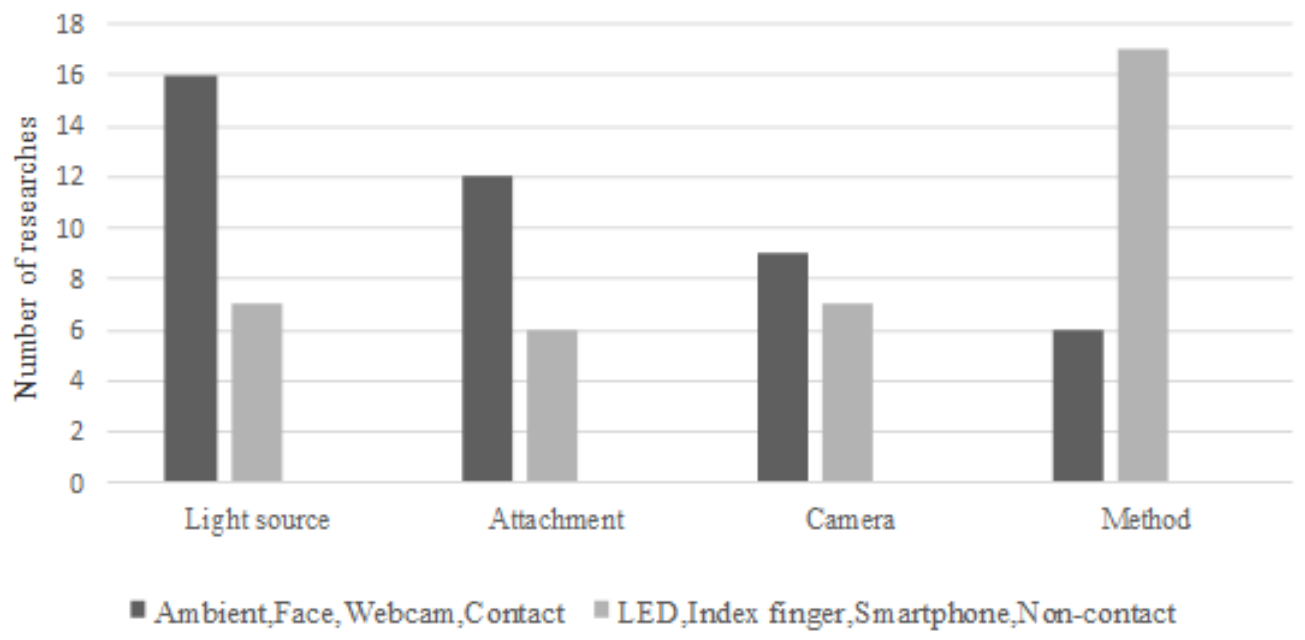

Fig. 2. Number of researches based on different parameters.

means photoplethysmography ("plethysmo" means "enlargement" in Greek). PPG is an easy and economical visual biomonitoring procedure used to noninvasively measure the blood volume changes. The changes occur in the microvascular tissue bed under the skin [32]. IPPG is based upon the PPG technology. It is a noncontact method and by means of tangential blood perfusion measurements it can identify heart generated pulse waves. The transmission or reflectance of light is affected by the variations in blood volume (i.e. in systole and diastole). Contact and noncontact PPG are two concepts of PPG to monitor the heart rate. PPG is used to monitor $\mathrm{HR}$ and $\mathrm{SpO} 2$ using webcam [8]. Under both indirect sun light and fluorescent light this method provides satisfactory accuracy. PPG is also used in other methods [9]. It's accuracy of estimation is increased. Some methods work based on PPG and takes conceivable minimum time and cost [21].

\section{G. Light source and Attachment}

Ambient light has been used as the light source in 16 research works of the 23 that we studied. LED has been used in the remaining 7 . Heart rate monitoring methods provide satisfactory results using ambient light, but in some methods by using LED we get more accurate results. Face has been used in 12 research works of 18 and index finger has been used in the remaining 6.9 of 16 researches use webcam and the remaining 7 use smartphone camera. In 23 research works that we studied 6 research works are contact methods and 17 works are non-contact. More precise results are provided by contact methods than non-contact methods but non-contact methods are more comfortable than contact methods. (see Fig. 2).

\section{CONCLUSION}

The number of heartbeats per unit of time is heart rate. Heart rate is represented as beat per minute (bpm). Heart rate specifies how well the heart is working. It can be a strong indicator of overall health and fitness. The traditional heart rate observing methods require physical contact. Many researchers have proposed many methods of heart rate measurement on their research articles which do not require any physical contact. These methods are more comfortable and easier to use w.r.t. the traditional methods.

In this study, we provide a comparative study on various heart rate monitoring methods using smartphone camera, webcam, different algorithms, smartphone apps, RGB components and PPG. These methods provide very promising results with satisfactory accuracy. These methods are uncomplicated, low cost, easy to implement and use. Works properly under different light conditions. Additional illumination increases the accuracy. The contact methods are more accurate and have high viability for heart rate monitoring than the non-contact methods.

These methods have some limitations. For some methods sufficient light on the facial region is required. A low brightness harms measurement quality. Any head motion in webcam-based methods affect the measurement quality. PPG measurements are very sensitive to subject's tissue. Some estimated algorithms will not work in all environmental conditions.

\section{REFERENCES}

[1] Kanva, Avneendra K., Chandan J. Sharma, and Sujay Deb. "Determination of SpO 2 and heart-rate using smartphone camera." In Proceedings of The 2014 International Conference on Control, Instrumentation, Energy and Communication (CIEC), pp. 237-241. IEEE, 2014.

[2] Peng, Rong-Chao, Xiao-Lin Zhou, Wan-Hua Lin, and Yuan-Ting Zhang. "Extraction of heart rate variability from smartphone photoplethysmograms." Computational and mathematical methods in medicine 2015 (2015).

[3] Yadav, Umang. "Photoplethysmography based Heart Rate Calculation using Fingertip Video Captured by Smartphone." (2016).

[4] Chen, Jie, Zhuoqing Chang, Qiang Qiu, Xiaobai Li, Guillermo Sapiro, Alex Bronstein, and Matti Pietikäinen. "RealSense= real heart rate: Illumination invariant heart rate estimation from videos." In 2016 Sixth International Conference on Image Processing Theory, Tools and Applications (IPTA), pp. 1-6. IEEE, 2016.

[5] Jain, Monika, Sujay Deb, and A. Venkata Subramanyam. "Face video based touchless blood pressure and heart rate estimation." In 2016 IEEE 18th International Workshop on Multimedia Signal Processing (MMSP), pp. 1-5. IEEE, 2016.

[6] Sanyal, Shourjya, and Koushik Kumar Nundy. "Algorithms for monitoring heart rate and respiratory rate from the video of a user's face." IEEE Journal of translational engineering in health and medicine 6 (2018): 1-11. 
[7] Xu, Shuchang, Lingyun Sun, and Gustavo Kunde Rohde. "Robust efficient estimation of heart rate pulse from video." Biomedical optics express 5, no. 4 (2014): 1124-1135.

[8] Bal, Ufuk. "Non-contact estimation of heart rate and oxygen saturation using ambient light." Biomedical optics express 6, no. 1 (2015): 86-97.

[9] Monkaresi, Hamed, Rafael A. Calvo, and Hong Yan. "A machine learning approach to improve contactless heart rate monitoring using a webcam." IEEE journal of biomedical and health informatics 18 , no. 4 (2013): 1153-1160.

[10] Muender, Thomas, Matthew K. Miller, Max V. Birk, and Regan L. Mandryk. "Extracting heart rate from videos of online participants." In Proceedings of the $2016 \mathrm{CHI}$ Conference on Human Factors in Computing Systems, pp. 4562-4567. 2016.

[11] Niu, Xuesong, Hu Han, Shiguang Shan, and Xilin Chen. "Continuous heart rate measurement from face: A robust rppg approach with distribution learning." In 2017 IEEE International Joint Conference on Biometrics (IJCB), pp. 642-650. IEEE, 2017.

[12] Qi, Huan, Zhenyu Guo, Xun Chen, Zhiqi Shen, and Z. Jane Wang. "Video-based human heart rate measurement using joint blind source separation." Biomedical Signal Processing and Control 31 (2017): 309-320.

[13] Yuvraj D. Patil \& Gyankamal J. C. "Heart Rate Measurement System using Facial Video." International Journal on Emerging Trends in Technology, vol. 4, no. 2, IJETT, 2017.

[14] Rahman, Hamidur, Mobyen Uddin Ahmed, Shahina Begum, and Peter Funk. "Real time heart rate monitoring from facial RGB color video using webcam." In The 29th Annual Workshop of the Swedish Artificial Intelligence Society (SAIS), 2-3 June 2016, Malmö, Sweden, no. 129. Linköping University Electronic Press, 2016.

[15] Addison, Paul S. "Modular continuous wavelet processing of biosignals: extracting heart rate and oxygen saturation from a video signal." Healthcare technology letters 3, no. 2 (2016): 111-115.

[16] Jindal, Vasu. "Integrating mobile and cloud for PPG signal selection to monitor heart rate during intensive physical exercise." In Proceedings of the International Conference on Mobile Software Engineering and Systems, pp. 36-37. 2016.

[17] Li, Xiaobai, Jie Chen, Guoying Zhao, and Matti Pietikainen. "Remote heart rate measurement from face videos under realistic situations." In Proceedings of the IEEE conference on computer vision and pattern recognition, pp. 4264-4271. 2014.

[18] Abe, Erika, Hiroshi Chigira, Koichi Fujiwarai, Toshitaka Yamakawa, and Manabu Kano. "Heart rate monitoring by a pulse sensor embedded game controller." In 2015 Asia-Pacific Signal and Information Processing Association Annual Summit and Conference (APSIPA), pp. 1266-1269. IEEE, 2015.

[19] Singh, Mridula, Abhishek Kumar, Kuldeep Yadav, Himanshu Madhu, and Tridib Mukherjee. "Mauka-mauka: measuring and predicting opportunities for webcam-based heart rate sensing in workplace environment." In Proceedings of the 10th EAI International Conference on Body Area Networks, pp. 96-102. 2015.

[20] Lam, Antony, and Yoshinori Kuno. "Robust heart rate measurement from video using select random patches." In Proceedings of the IEEE International Conference on Computer Vision, pp. 3640-3648. 2015.

[21] Patil, Yuvraj D., and Gyankamal J. Chhajed. "Heart Rate Measurement System using Facial Video Processing." IJETT 1, no. 2 (2017).

[22] Rouast, Philipp V., Marc TP Adam, Raymond Chiong, David Cornforth, and Ewa Lux. "Remote heart rate measurement using lowcost RGB face video: a technical literature review." Frontiers of Computer Science 12, no. 5 (2018): 858-872.

[23] Procházka, Aleš, Martin Schätz, Oldřich Vyšata, and Martin Vališ "Microsoft kinect visual and depth sensors for breathing and heart rate analysis." Sensors 16, no. 7 (2016): 996.

[24] Peng, Rong-Chao, Wen-Rong Yan, Ning-Ling Zhang, Wan-Hua Lin, Xiao-Lin Zhou, and Yuan-Ting Zhang. "Investigation of five algorithms for selection of the optimal region of interest in smartphone photoplethysmography." Journal of Sensors 2016 (2016).

[25] Coppetti, Thomas, Andreas Brauchlin, Simon Müggler, Adrian Attinger-Toller, Christian Templin, Felix Schönrath, Jens Hellermann, Thomas F. Lüscher, Patric Biaggi, and Christophe A. Wyss. "Accuracy of smartphone apps for heart rate measurement." European journal of preventive cardiology 24, no. 12 (2017): 1287-1293.
[26] Alghoul, Karim, Saeed Alharthi, Hussein Al Osman, and Abdulmotaleb El Saddik. "Heart rate variability extraction from videos signals: ICA vs. EVM comparison." IEEE Access 5 (2017): 4711-4719.

[27] Papon, Md Tarikul Islam, Ishtiyaque Ahmad, Nazmus Saquib, and Ashikur Rahman. "Non-invasive heart rate measuring smartphone applications using on-board cameras: A short survey." In 2015 International Conference on Networking Systems and Security (NSysS), pp. 1-6. IEEE, 2015.

[28] Adibuzzaman, Mohammad, Sheikh Iqbal Ahamed, and Richard Love. "A personalized model for monitoring vital signs using camera of the smart phone." In Proceedings of the 29th Annual ACM Symposium on Applied Computing, pp. 444-449. 2014.

[29] Verkruysse, Wim, Lars O. Svaasand, and J. Stuart Nelson. "Remote plethysmographic imaging using ambient light." Optics express 16, no. 26 (2008): 21434-21445.

[30] Jonathan, E., and Martin Leahy. "Investigating a smartphone imaging unit for photoplethysmography." Physiological measurement 31, no. 11 (2010): N79.

[31] Allen, John. "Photoplethysmography and its application in clinical physiological measurement." Physiological measurement 28, no. 3 (2007): R1.

[32] Sun, Yu, and Nitish Thakor. "Photoplethysmography revisited: from contact to noncontact, from point to imaging." IEEE Transactions on Biomedical Engineering 63, no. 3 (2015): 463-477.

[33] Kirk, Sarah E. "Comparison of the Apple Watch, Fitbit Surge, and Actigraph GT9X Link in Measuring Energy Expenditure, Steps, Distance, and Heart Rate." PhD diss., Cleveland State University, 2016.

[34] Kwon, Sungjun, Hyunseok Kim, and Kwang Suk Park. "Validation of heart rate extraction using video imaging on a built-in camera system of a smartphone." In 2012 Annual International Conference of the IEEE Engineering in Medicine and Biology Society, pp. 2174-2177. IEEE, 2012.

[35] Datcu, Dragos, Marina Cidota, Stephan Lukosch, and Leon Rothkrantz. "Noncontact automatic heart rate analysis in visible spectrum by specific face regions." In Proceedings of the 14th International Conference on Computer Systems and Technologies, pp. 120-127. 2013.

[36] Holton, Benjamin D., Kavan Mannapperuma, Peter J. Lesniewski, and John C. Thomas. "Signal recovery in imaging photoplethysmography." Physiological measurement 34, no. 11 (2013): 1499. 